\section{Cureus}

\title{
Pediatric Motor Vehicle-Pedestrian Accident: a Simulation Scenario for Emergency Medicine Trainees
}

\author{
Sarah Mathieson ${ }^{1}$, Kerry-Lynn Williams ${ }^{2}$, Adam Dubrowski ${ }^{3}$ \\ 1. Emergency Medicine, Memorial University of Newfoundland 2. Medical Education and Simulation, \\ Memorial University of Newfoundland, St. John's, CAN 3. Health Sciences, University of Ontario Institute \\ of Technology, Oshawa, CAN
}

$\square$ Corresponding author: Kerry-Lynn Williams, kwilliams@mun.ca

Disclosures can be found in Additional Information at the end of the article

\section{Abstract}

Simulation-based medical education is an evolving field that allows trainees to practice skills in a safe environment with no risk to patients. Recently, technology-enhanced simulation for emergency medicine learners has been shown to have favorable effects on learner knowledge and patient outcomes. In this report, a human patient simulator is used to familiarize emergency medicine trainees with the presentation and management of a pediatric motor vehicle-pedestrian accident is described.

\section{Categories: Emergency Medicine, Medical Simulation, Pediatrics}

Keywords: emergency medicine, pediatric emergency medicine, simulation-based medical education, pediatric trauma

\section{Introduction}

Trauma is the leading cause of death and disability in children greater than one year of age [1]. Despite this fact, it is still a relatively uncommon event and many trainees do not get adequate exposure to it without the use of simulation [2]. Much of the available literature indicates that the repeated use of high-fidelity simulation can improve the acquisition of knowledge, skills, and attitudes, as well as their translation to the clinical setting in relation to neonatal and pediatric resuscitation, Cardiopulmonary resuscitation (CPR) and procedural skills, including airway management [3-4]. "Procedural skills that used to be taught with the "see one, do one, teach one" approach are now being taught with a safer approach: "see one, simulate a lot, do one, teach one” [4].

Received 10/20/2016 Review began 10/27/2016 Review ended 02/21/2017 Published 02/23/2017

\section{(c) Copyright 2017}

Mathieson et al. This is an open access article distributed under the terms of the Creative Commons Attribution License CC-BY 3.0., which permits unrestricted use, distribution, and reproduction in any medium, provided the original author and source are credited.
Pediatric trauma can present the emergency room physician with challenges that are unique compared to adult trauma. Firstly, trauma in the pediatric setting is emotionally charged. Secondly, due to differences in anatomy and physiology in children compared with adults, injury management also differs in some regards. For a physician not working primarily in a pediatric trauma center, these differences may add an element of discomfort when dealing with the injured child. Although many injuries can be managed initially in a general emergency department, more seriously injured children require transportation to a designated pediatric trauma center.

The use of simulation can help to familiarize residents, as well as practicing physicians, with the various elements of pediatric trauma, thereby improving performance in real-life situations. Simulation is a useful tool to improve medical skills and knowledge. It also addresses 


\section{Cureus}

the less tangible aspects of running a trauma; for example teamwork [5], dealing with death or significant morbidity and emotional reaction of the healthcare team. This is especially important in the pediatric setting.

\section{Technical Report}

This in-situ simulation training session was conducted in the emergency department at the Janeway Children's Health and Rehabilitation Centre using a high-fidelity mannequin simulator. This particular simulation used Laerdal SimJunior ${ }^{\mathrm{TM}}$ (232-05050).

Prior to the session, a comprehensive stepwise scenario template was developed (Table 1). This process required compiling all of the relevant clinical data and supporting documents, such as $\mathrm{x}$-rays (Figures 1,2) and laboratory data, that were to be used during the scenario execution. This was submitted to the simulation laboratory technical staff who then programmed the mannequin and supplied any necessary materials for the simulation.

Table 1: A stepwise, detailed scenario template that was submitted to the simulation lab's technical staff, who then programmed the mannequin and supplied the necessary materials for the case.

\section{Pre-Scenario}

You are working in a rural emergency department. An eight-year-old male is brought in by ambulance after being struck by a pick-up truck as he ran across the road. He has fractures to his left femur and right elbow as well as various abrasions. The nearest trauma center is 90 minutes by road.

History

Allergies Peanuts

Medications $\quad$ Flovent $^{\mathrm{TM}}$, prn Ventolin ${ }^{\mathrm{TM}}$

Past Medical Hx Asthma (mild)

T 36.5 (axillary) // HR 120 (sinus) // BP 100/65 // RR24 // spO2 100\% RA // glucose 6 (as per EMS)

Initial Vitals The patient is alert, crying, anxious and in pain, doesn't want to talk to anyone but his father; collared and boarded by EMS

HEENT Laceration to R cheek

CNS Normal apart from anxious appearance

Chest

Heart sounds normal, breath sounds normal bilaterally, trachea midline no obvious contusions or deformity

Abdomen Soft; non-tender

Extremities

Deformed $L$ femur, open wound; swollen and deformed $R$ elbow; abrasions to $R$ hip and arm; contusions to $L$ hip and thigh

Objective 1: Develop an approach to pediatric trauma

Stage 1: Initial Assessment / Stabilization

Stage

Vitals and Results of Investigations

Expected Action 


\section{Cureus}

\section{Connect to}

cardiac monitor,

oxygen

saturation

monitor, get new

set of vitals,

place 2 large

bore IVs, assess

ABCDE

Get new set of

vitals

A - airway patent and protected B - RR 24, no respiratory distress, clear air bilaterally, trachea midline C - BP 100/65, HR 120, heart sounds normal, peripheral pulses strong $x 4$ limbs, abdomen soft and non-tender, obvious deformity and overlying open wound to $L$ femur $D$ - alert and oriented $x 3$, power/tone/sensation/reflexes normal $x 4$ limbs, pupils equal and reactive $E$ undress and logroll child while maintaining c-spine precautions to remove backboard and examine spine

\section{Without fluid} resuscitation

\section{Same as EMS}

Start two large bore IVs

$H R$ increases to 130

With father's

presence

$\mathrm{RR}$ to 16 , child calmer

If adequate fluid Vitals stable

HR 135 // BP 85/50

If inadequate

fluid

HR $135 / / \mathrm{BP} 85 / 50$

Pain control should be given, second bolus given. After second bolus, should consider $\mathrm{O}^{-}$blood if not stable

Continue to stage two

Continue to stage two

$\mathrm{CBC}$, electrolytes,

BUN, creatinine, liver

Order

investigations

and labs

Vitals stable enzymes, amylase, coagulants, T\&S, crossmatch), x-rays (CXR, pelvis, c-spine, $L$ femur and $R$ elbow)

Stage 2: Mom arrives, visibly upset and agitated

Mom addressed

by lead and

promptly

assigned a

Continue on to stage

chaperone

(nurse or other)

No one promptly addresses

Mom's anxiety

Child's anxiety increases. HR increases by $5 /$ min, RR 22, the child starts crying again three

Address Mom, and then continue on to stage three

Objective 2: Recognize common pediatric injuries and their appropriate management

Stage 3: Secondary Survey

Secondary 


\section{Cureus}

survey

completed,

FAST, X-ray c-

spine, L leg, $R$

elbow, CT, EKG

Results of

ordered tests
Found to have a $\mathrm{L}$ mid-shaft femur fracture and a $\mathrm{R}$ supracondylar fracture. No intra-abdominal fluid on FAST. No chest or intra-abdominal injury on CT. Laceration that requires suturing to $\mathrm{R}$ cheek. Abrasions to $\mathrm{R}$ hip and arm, contusions to L hip and thigh. No other injury. EKG unremarkable

hemoglobin 116 hematocrit 0.45 platelets 250 white blood cell count 7.0 glucose $4.0 \mathrm{Na} 135 \mathrm{Cl} 90 \mathrm{~K} 4.0 \mathrm{CO} 224$ AST 35 ALT 40 ALP 70 total bilirubin 16 amylase 70 INR 1.0 PTT $40 \mathrm{sec}$ blood type A+

$\mathrm{R}$ arm); antibiotics and tetanus. Take measures to keep temperature physiologic

Get new set of vitals

Objective 3: Prepare for transport to a trauma center

Stage 4: Prepare for transport

Warm blankets, dressing to $\mathrm{R} \quad$ Vitals stable cheek

If not adequate fluids

HR 140 // BP 75/50

HR 150 // BP 60/35 Child becomes drowsy
Call surgeon and arrange transport to trauma centre.

Consider having a unit of packed red blood cells available during transport

Stabilize child then arrange transport to trauma centre.

Consider having a unit of packed red blood cells available during transport

Stabilize child then arrange transport to trauma centre.

Consider having a unit of packed red blood cells available during transport

TABLE 1: A stepwise, detailed scenario template was submitted to the simulation lab's technical staff, who then programmed the mannequin and supplied the necessary materials for the case 


\section{Cureus}

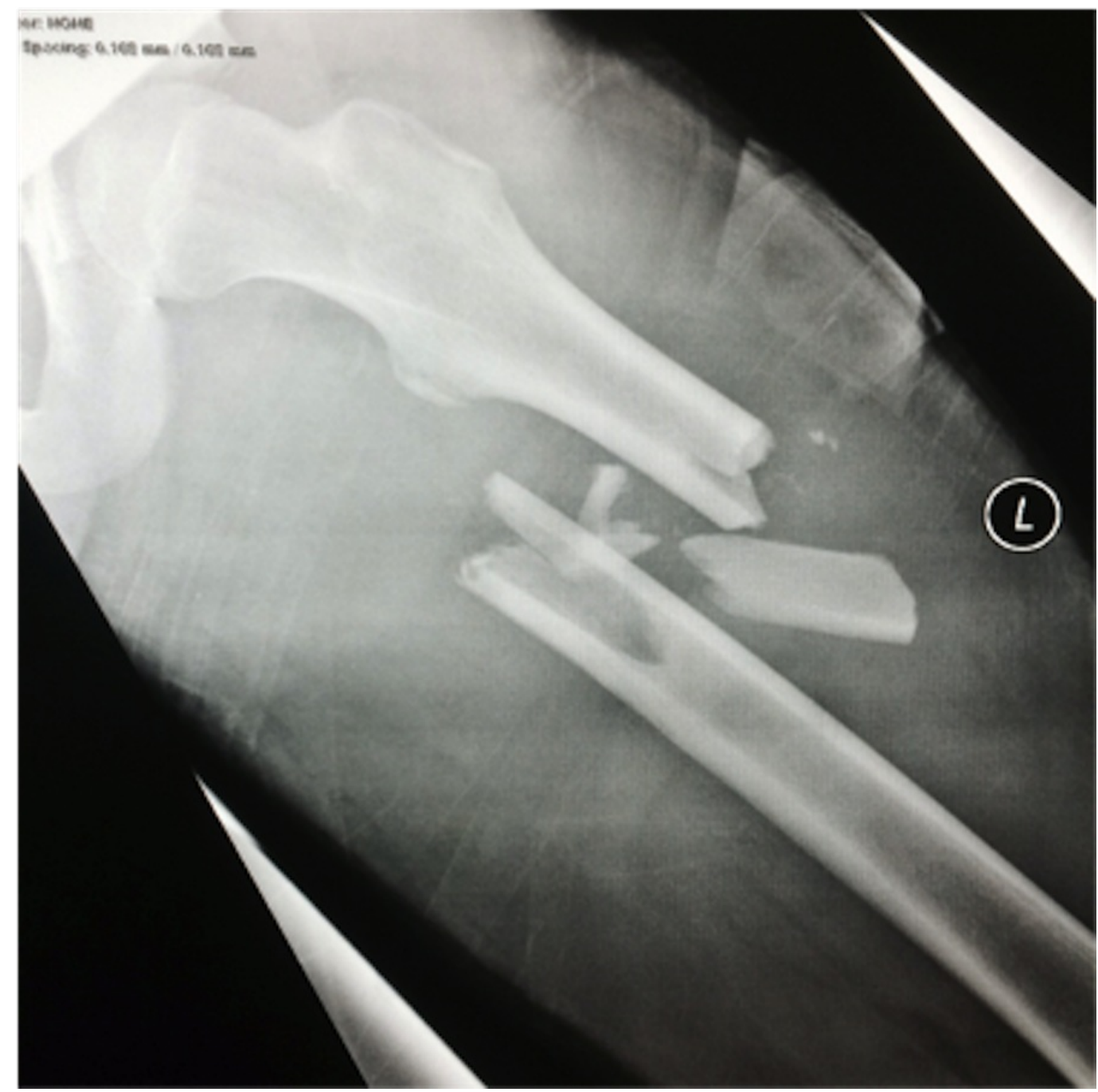

FIGURE 1: Radiograph of left femur demonstrating a comminuted mid-shaft fracture (Source: K Chan)

Figure 1: Radiograph of left femur demonstrating a mid-shaft fracture (Source: K. Chan)
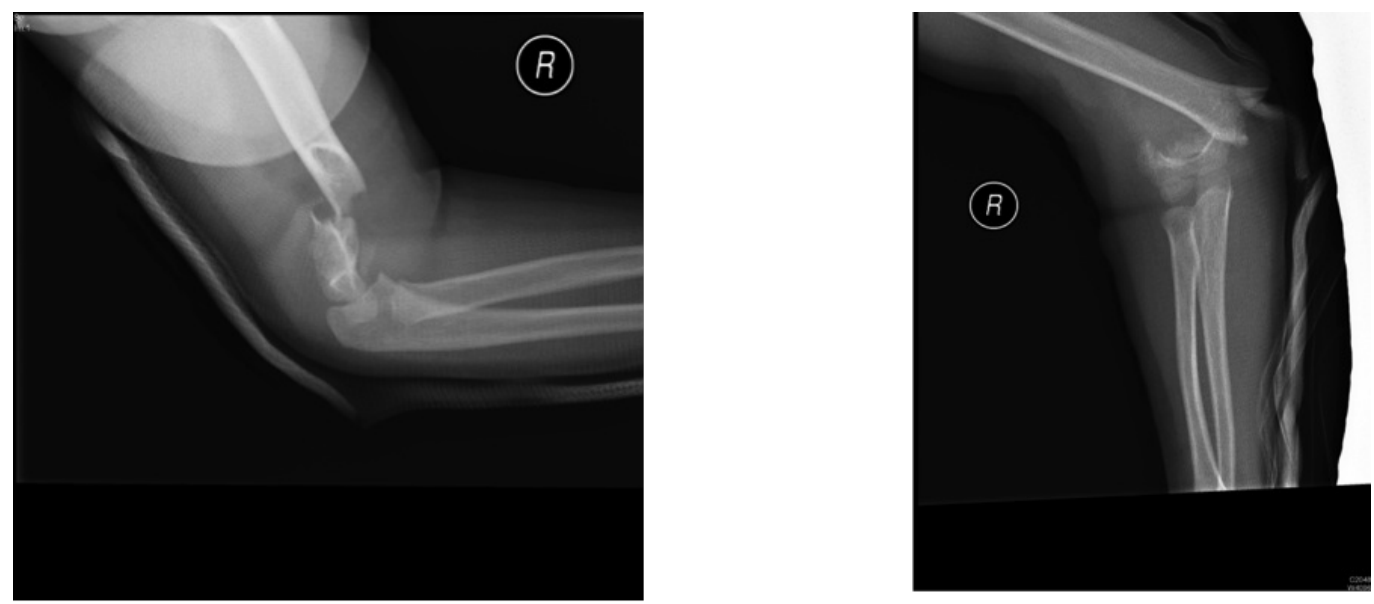

FIGURE 2: Radiographs of the right elbow demonstrating a 
Figure 2: Radiographs of the right elbow demonstrating a supracondylar fracture (Source: K. Chan)

To ensure a smooth experience for trainees, an instructor completed a run-through of the scenario while acting as a trainee, prior to formal learning sessions. During a learning session, two instructors were present, one who maintained overall control of the scenario, and the second who took notes for the subsequent debriefing.

Pre-Briefing

A pre-briefing was held with the trainees before the case. Limitations of the simulation were reviewed, in particular addressing technical issues with the mannequin and resource availability. The fiction contract agreement between participants and instructors to proceed as if the simulation is real while, simultaneously acknowledging it was not addressed [6]. Finally, trainees were advised that the case is strictly formative.

Case

In this simulation case, an eight-year-old male is brought to a community hospital after being struck by a pick-up truck as he ran across the road. His father, who accompanied the boy in the ambulance, saw the accident and said he did not think the boy lost consciousness. Upon request, trainees are provided with details of the patient's allergies, medications, and a past medical history that includes mild asthma. Learners were advised if an investigation was normal and/or were provided with a normal result from the simulation laboratory database.

At the beginning of the scenario, the patient is already connected to cardiac monitors with a full set of vital signs provided by emergency medical services (EMS) indicating an axillary temperature of $36.5^{\circ} \mathrm{C}$, tachycardia, and a blood pressure of $100 / 65$. The scenario takes place in the trauma room of a community hospital with a full complement of resuscitation and intubation equipment on hand, as well as point of care ultrasound (PoCUS). Also available are crystalloid or colloid fluids, blood $\left(\mathrm{O}^{-}\right.$and type-specific), a rapid infuser, warming devices, splints (including Thomas), lab, x-ray, and computed tomography (CT). There are two registered nurses as well as a respiratory therapist working, and there is a general surgeon on call at the nearest trauma center.

The trainees are then instructed to proceed with their evaluation of the patient.

Debriefing

Following the conclusion of the scenario, trainees were provided with a formal debriefing. Care was taken during the debriefing to ensure that the number of debriefers is limited such that the debriefer-to-learner ratio does not exceed one: one. The debriefing is ideally lead by an educator with experience in debriefing; the principles of good judgment and frame discovery should be central to the process [7].

\section{Post-Scenario Didactics}

A didactic session was held after the debriefing. During this session, instructors were able to 
address any learning needs identified through the scenario and debriefs, and trainees were given the opportunity to strengthen knowledge gained through the simulation exercise. The following is meant to be a guide only, not a complete discussion.

Objective 1: Develop an approach to pediatric trauma: A general approach to trauma as outlined by the advanced trauma life support (ATLS) guidelines should be reviewed [8]. This provides the learner with a systematic, routine approach that prioritizes life and then limb threatening injuries. A thorough secondary survey can be conducted once the primary survey is complete and any life and limb threatening injuries are stabilized appropriately. Special consideration should be given to the mechanism of injury and the possible resulting injuries. In this case, suspicion for a femur fracture is high as this is common for children involved in an multi-voxel pattern analysis (MVPA). Chest and abdominal trauma must also be ruled out in any child hit by a vehicle [9]. It is also important for learners to recognize that children are likely to be reassured by the presence of a parent or caregiver and that this will diffuse some fear, enabling easier assessment and management of injuries. Of course, a parent or substitute decision maker is required to be involved for informed consent when emergent care will not be delayed to obtain consent.

Objective 2: Recognize common pediatric injuries and their appropriate management: Although the basic tenets of trauma are the same for adults and pediatrics, special consideration should be given to a few differences. For example, c-spine injury patterns vary in young children due to anatomical differences; chest wall damage may be minimal despite significant pulmonary or cardiac injury due to rib cage compliance; abdominal solid organ injury is more likely in children in the setting of blunt trauma [9]. The educator should be familiar with pediatric trauma and therefore be able to highlight any knowledge gaps amongst the learners.

Objective 3: Prepare for transport to a trauma center: The logistics of transportation from a regional center to a tertiary trauma center will vary from center to center. However, certain principles apply to all situations. Distance to the trauma center and the time required to mobilize transportation should be considered. Is ground or air transport available? Which is more appropriate to the geography, as well as to the urgency of the patient's condition? It is important to ensure that staff trained to manage the severity of the patient's injuries are available to accompany the patient. It is also important to ensure measures are taken to reduce morbidity or mortality during transport. For example, a patient may be electively intubated prior to transport if deterioration of the airway is anticipated during transport. The open femur fracture should be cleaned and dressed with minimal handling and then splinted before transport. The elbow fracture should also be splinted before transport. Also, fluid resuscitation, blood products, and analgesia should be available during transport. Ground transport via ambulance is a reasonable choice in this case, though a case could be made for air transport if the service cab can get the patient to the trauma center in less than 90 minutes. A physician escort is ideal, however, if no other physician in the community is available, royal navy (RN) escort would also be acceptable. A parent should accompany the child.

These issues should be discussed with the learner by the physician leading the didactic session. It is expected the learners are able to recognize when transport is necessary, and what preparation is required.

\section{Discussion}

Teaching emergency medicine trainees how to manage pediatric trauma and prepare for transport to a designated pediatric trauma center through the use of a case simulation may be a valuable teaching tool. 
In this simulation, key learning objectives for trainees include:

1. Develop an approach to pediatric trauma,

2. Recognize common pediatric injuries and their appropriate management,

3. Considerations for transport to a trauma center.

During the post-scenario didactic session, we considered the mechanism of injury and the importance of a thorough secondary survey. The logistics of transport was discussed.

The development of the case scenario using a stepwise algorithm allows the simulation to react according to decisions made by trainees. Having an instructor complete the run-through both ensures that the case is of reasonable difficulty for the resident and enables instructors to address any limitations of the scenario. Finally, the use of a formal debriefing model coupled with a post-scenario didactic session allows instructors to identify and address not only knowledge gaps but also errors of process committed by trainees.

\section{Conclusions}

Pediatric trauma can be very challenging and emotionally charged. Therefore, training in a controlled environment can be beneficial for postgraduate trainees and ultimately lead to better patient outcomes. We have presented a pediatric motor vehicle-pedestrian simulation scenario. An integrated teaching session incorporating simulation and didactics with components of debriefing targeted toward postgraduate medical learners is also presented.

\section{Additional Information}

\section{Disclosures}

Human subjects: All authors have confirmed that this study did not involve human participants or tissue. Animal subjects: All authors have confirmed that this study did not involve animal subjects or tissue. Conflicts of interest: In compliance with the ICMJE uniform disclosure form, all authors declare the following: Payment/services info: All authors have declared that no financial support was received from any organization for the submitted work. Financial relationships: All authors have declared that they have no financial relationships at present or within the previous three years with any organizations that might have an interest in the submitted work. Other relationships: All authors have declared that there are no other relationships or activities that could appear to have influenced the submitted work.

\section{Acknowledgements}

This project was supported by the Tuckamore Simulation Research Network and Emergency Medicine Educational Committee, Memorial University. We would also like to acknowledge contributions from the Janeway Foundation and Dr. Kevin Chan, Chair of the Discipline of Pediatrics, Faculty of Medicine, Memorial University of Newfoundland.

\section{References}

1. National centre for health statistics: Health, United States, 2007: chartbook on trends in the health of Americans. US Department of Health and Human Services, Hyattsville, MD; 2007.

2. Nadel FM, Lavalle JM, Fein JA, Giardino AP, Decker JM, Durbin DR: Assessing paediatric senior residents training in resuscitation: fund of knowledge, technical skills and perception of confidence. Pediatr Emerg Care. 2000, 16(2):73-6.

3. Ilgen JS, Sherbino J, Cook DA: Technology-enhanced simulation in emergency medicine: a 


\section{Cureus}

systematic review and meta-analysis. Acad Emerg Med. 2013, 20(2):117-127.

10.1111/acem.12076

4. Lin Y, Cheng A: The role of simulation in teaching pediatric resuscitation: current perspectives. Adv Med Educ Pract. 2015, 6:239-48. 10.2147/AMEP.S64178

5. Bank I, Snell L, Bhanji F: Pediatric crisis resource management training improves emergency medicine trainees perceived ability to manage emergencies and ability to identify teamwork errors. Pediatr Emerg Care. 2014, 30(12):879-83. 10.1097/PEC.0000000000000302

6. The contract essential to the parties of simulation . (2015). Accessed: September 4, 2016: https://simulatinghealthcare.net/2015/05/06/the-contract-essential-to-the-parties-ofsimulation/.

7. Rudolph JW, Simon R, Rivard P, Dufresne RL, Raemer DB: Debriefing with good judgement: combining rigorous feedback with genuine inquiry. Anesthesiol Clin. 2007, 25(2):361-76. 10.1016/j.anclin.2007.03.007

8. American college of surgeons committee on trauma: ATLS advanced trauma life support program for doctors, 9th ed. American College of Surgeons, Chicago, IL; 2012.

9. Marx JA: Rosen's emergency medicine: concepts and clinical practice, 6th ed. Mosby Elsevier, Philadelphia, PA; 2006. 